\title{
High energy emission from the Galactic Center: Theory and observations
}

\author{
M. Chernyakova ${ }^{1,2, a}$, D. Malyshev ${ }^{3, b}$, A. Neronov ${ }^{3}$, and R. Walter ${ }^{3}$ \\ 1 ISDC Data Centre for Astrophysics \\ 2 Dublin City University \\ 3 Dublin Institute for Advanced Studies
}

\begin{abstract}
Galactic Centre is a bright $\gamma$-ray source with the $\mathrm{GeV}-\mathrm{TeV}$ band spectrum composed of two distinct components in the $1-10 \mathrm{GeV}$ and $1-10 \mathrm{TeV}$ energy ranges. The nature of the two components is not clearly understood. We report the analysis of the data of 74 months of observations of the Galactic Center by Fermi/LAT $\gamma$-ray telescope with the goal to constrain the morphology of the source and the nature of the two components. Spatially the Galactic Center is consistent with a point source with $0.13^{\circ} 3 \sigma$ upper limit on its radius. We use the Fermi/LAT data in the energy band below $100 \mathrm{MeV}$ to show that the $\gamma$-ray emission from the Galactic Centre source can't be explained within the pure hadronic model and discuss the necessary modifications to make the model to be consistent with the observational data. We also discuss an alternative self-consistent interpretation of the $60 \mathrm{MeV}-30 \mathrm{TeV}$ spectrum of the source by a model in which the signal is produced via inverse Compton scattering of the ambient infrared radiation field.
\end{abstract}

\section{Introduction}

The Galactic Centre (GC) is a unique astronomical source hosting the nearest supermassive black hole of the mass $M \simeq 4 \times 10^{6} M_{\odot}$ accreting matter in a radiatively inefficient regime and producing infrared, X-ray and $\gamma$-ray emission with luminosity $L \simeq 10^{36} \mathrm{erg} / \mathrm{s}$ (see e.g. [1] for a review).

The X-ray and infrared source could be associated to the supermassive black hole because of its variable nature.

To the contrary, the source of the GeV and $\mathrm{TeV} \gamma$-rays is not variable and it is not clear if the emission comes from the vicinity of the black hole or it is produced in an extended region around the black hole or, finally, it comes from an unrelated source positionally coincident with the supermassive black hole in Sgr A*. In this paper we report a study of the spectral, timing and imaging characteristics of the GC source in the energy range $60 \mathrm{MeV}-300 \mathrm{GeV}$ using 6 years of observations with Fermi/LAT. We discuss implications of our findings for the various theoretical models.

\section{Data analysis and results}

In our work we use 74 months of Fermi/LAT data (from August, 4th, 2008 to September, 25th, 2014). We perform the binned likelihood analysis of the data using the most recent Fermi Science Tools Software v9r33p0 and the P7REP response functions. In this work we also use INTEGRAL/PICSIT data obtained from February 2003 to August 2012.The details of the data analysis is given in [2].

a e-mail: masha. chernyakova@dcu.ie

b e-mail: Denys.Malyshev@unige.ch

\subsection{The morphology of the source.}

At high energies $(\gtrsim 10 \mathrm{GeV})$ the Fermi/LAT PSF is quite narrow $\left(\sim 0.2^{\circ} 68 \%\right.$ PSF containment). At these energies we test the hypothesis of the diffuse nature of the GC discussed in the literature. To do this we replace the GC point-like source with a uniform disk. We first perform the fitting procedure for the model without the central source (GC), and afterwards we add to the model the GC disk with radius varying in the $0.02^{\circ}-0.3^{\circ}$ range. The TS of the disk is the difference between log-like values of these models [seee.g. 3]. This procedure is similar to the one described in [4] for the localization of the catalogue sources. For small radii the GC is detected with high TS values, $T S=178$, which corresponds to $\sim 13 \sigma$ statistical significance, see Fig. 1.

We find that the TS remains roughly the same for disk radii $\lesssim 0.1^{\circ}$ and rapidly decreases at $r \gtrsim 0.13^{\circ}$. At this radius the TS decreases to 169 . This source extension corresponds to the $3 \sigma$ upper limit on the source size.

The flux attributed to the GC source for radii $r<0.13^{\circ}$ remains constant within a $10 \%$ systematic error, see Fig. 1, right. This allows us to consider the GC as a point source for the spectral analysis. The same procedure, performed at a lower $3-10 \mathrm{GeV}$ energy band gives similar results. The significance of the added disk does not change for radii $\lesssim 0.15$, with TS dropping down by $9(3 \sigma)$ at $r \approx 0.22^{\circ}$.

\subsubsection{FermilLAT spectral analysis}

The spectral model of each point source in the region of interest is chosen to be a powerlaw with the slope -2 in

\footnotetext{
${ }^{1}$ Note, that this size is similar to the distance $\left(0.093^{\circ}\right)$ between the GC and the closest to it source in 3FGL catalog.
}

This is an Open Access article distributed under the terms of the Creative Commons Attribution License 4.0, which permits unrestricted use, distribution, and reproduction in any medium, provided the original work is properly cited. 

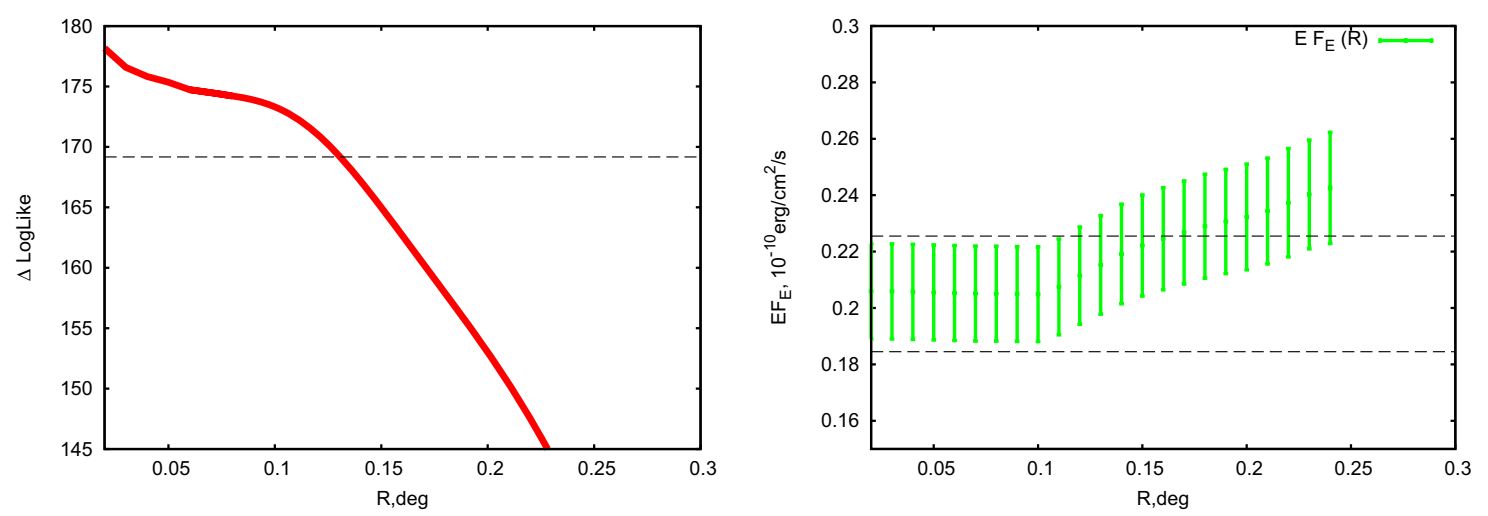

Figure 1. We model the GC as a disk in the $10-300 \mathrm{GeV}$ energy band. Left panel: significance of added source as a function of disk radius. Dashed lines show $3 \sigma$ confidence range for the source size. Right panel: best-fit value of the flux corresponds to certain disk radius. $10 \%$ systematics level is shown with dashed black lines.
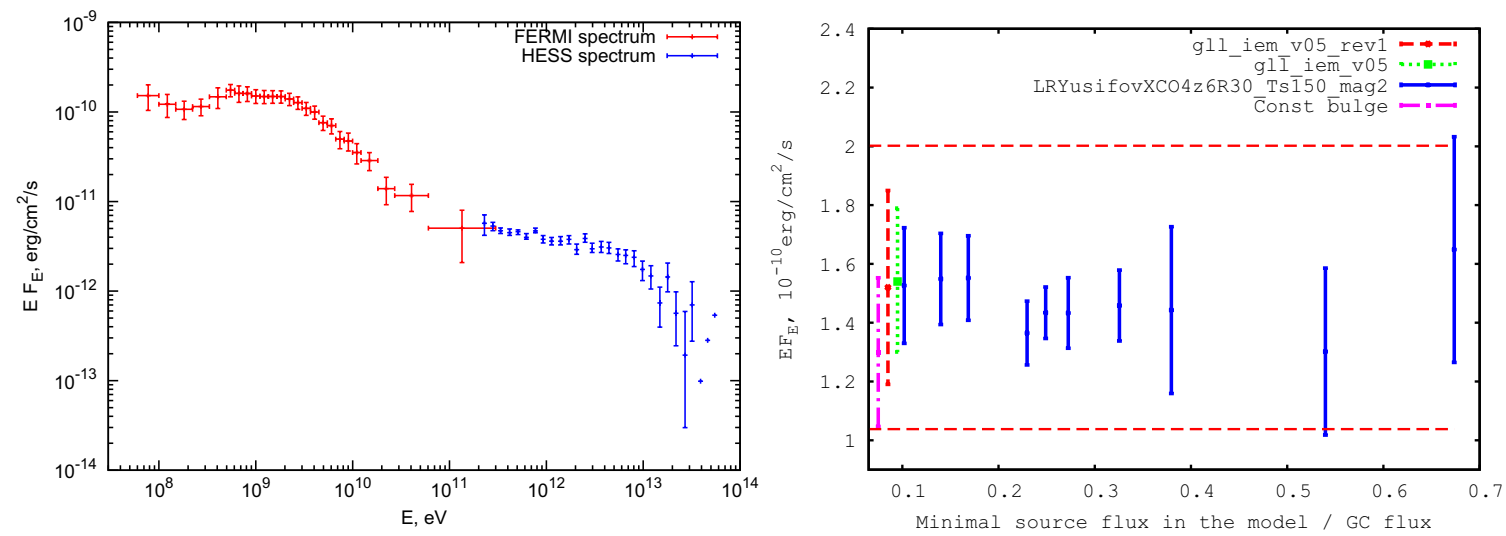

Figure 2. Left panel: combined Fermi/LAT -HESS spectrum of the Galactic Centre. 10\% systematics was added to Fermi data points. Right panel: the flux in $60-100 \mathrm{MeV}$ energy bin as a function of minimal catalog flux of the sources included into the model of the galactic diffuse emission). The dashed horizontal lines indicate the flux errorbars in $60-100 \mathrm{MeV}$ band from the left panel.

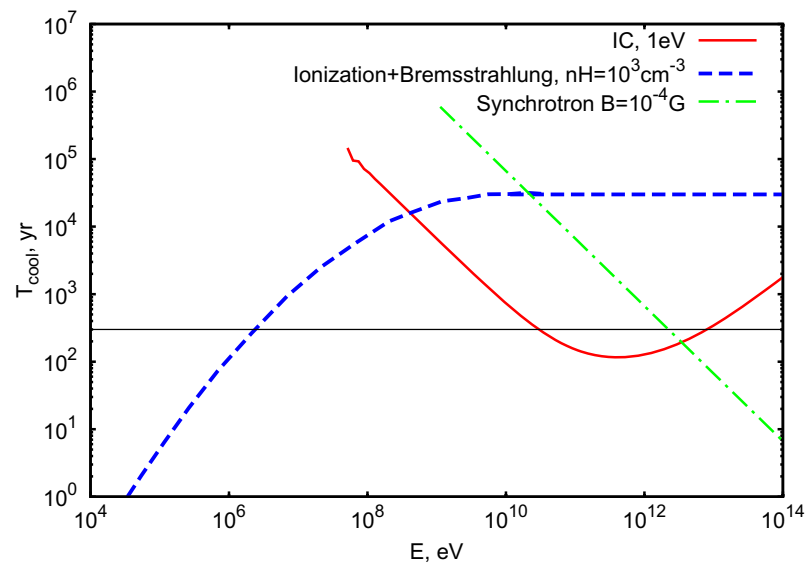

Figure 3. Electron energy loss cooling times for the medium parameters similar to observed in the vicinity of the GC. The timescale of $300 \mathrm{yrs}$ corresponding to the flaring activity age is shown with thin solid line for the convenience.

each energy bin. The diffuse background model spectra are following the templates, the iso_source_v05_rev1.txt for the isotropic background and several different templates for the Galactic diffuse emission background. The resulting spectrum of the source is showing in Fig. 2.
In addition to statistical errors, we include a $10 \%$ systematic error in the energy range $100 \mathrm{MeV}-1 \mathrm{TeV}$ [5]. This systematic error characterises uncertainties of the knowledge of characteristics of the LAT telescope. Taking into account the complexity of the GC region, we perform a separate study of additional systematic error stemming from the uncertainties of the knowledge of the properties of the interstellar medium (and as a consequence, of the diffuse Galactic emission) and of the point source distribution in the region of interest. We do this by repeating the analysis using several templates for Galactic diffuse emission and several sets of point sources. We verify that all the used models give fluxes of the GC consistent within $10 \%$. Changing the limiting flux level for the catalog sources, or changing the catalog altogether from 2FGL to 3FGL also produces changes in the flux which are within the $10 \%$ level, see Fig. 2, right panel.

\section{Discussion}

The origin of the multiwavelength emission from the Galactic Center has been extensively discussed in the literature, starting from the pre-Fermi epoch. These models use different types of radiation-producing particles, and majority of them can be divided into two broad classes, leptonic and hadronic. The spectrum of the GC shown in 

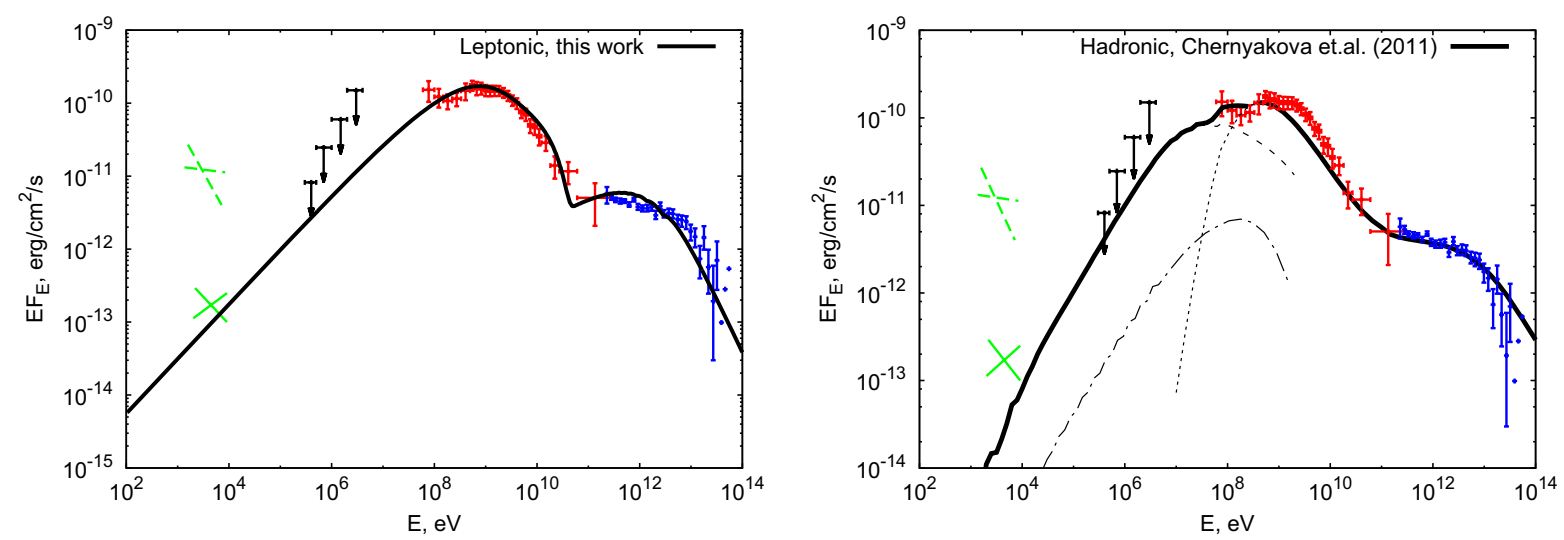

Figure 4. X-ray to TeV energies spectrum of the Galactic Centre. Left panel: the leptonic model proposed in this work for the low density case. Right panel: solid black line shows the hadronic model adopted from [7]. Pion decay emission component considered by authors is shown with thin dotted line. The absence of the low-energy cutoff in the observable Fermi/LAT data is in tension with purely hadronic origin of gamma-rays. Electrons bremsstrahlung radiation component (shown for proton to electron densities ratio $n_{p} / n_{e}=50$, dashed line) can significantly reduce this tension.

Fig. 2 extends to energies down to $60 \mathrm{MeV}$. This potentially opens a possibility to distinguish between the leptonic and hadronic models because the hadronic spectral models are generically expected to posses a spectral feature in the $100 \mathrm{MeV}$ energy range.

\subsection{Leptonic model}

The MeV-TeV spectrum of the GC can be interpreted with a simple one-zone leptonic model if one takes into account the flaring nature of the source. In this case the emission is a combination of the emission from electrons injected during the strong flare which occurred $\sim 300 \mathrm{yrs}$ ago [see e.g. 6, forthereview] and during recent much weaker activity. Electrons propagate through a medium with the density about $10^{3} \mathrm{~cm}^{-3}$, through the soft photon field with density $\sim 5 \times 10^{4} \mathrm{eV} / \mathrm{cm}^{-3}$ at $\sim 0.3-3 \mathrm{eV}$ and magnetic field of the order of $10-1000 \mu \mathrm{G}$. The main energy loss channels are ionisation, bremsstrahlung, inverse Compton (IC) and synchrotron mechanisms. Figure 3 shows a comparison of the cooling times for these energy loss channels, as a function of electron energy.

We find that the observed source spectrum could be well reproduced by models with rather different choice of parameters. Depending on the ambient medium density, the observed spectrum can be explained either with a pure IC model (low medium density, see left panel of Fig. 4), or by a combination of a IC scattering with a bremsstrahlung emission.

In the left panel of Fig. 4 we show an inverse Compton dominated model fit in which electrons are scattered on photons with energy $0.5 \mathrm{eV}$ and energy density $5 \times$ $10^{4} \mathrm{eV} / \mathrm{cm}^{3}$. The magnetic field is assumed to be $2.5 \times$ $10^{-4} \mathrm{G}$. Electrons are injected with a spectral index -1.5 for $10 \mathrm{yrs}$ during the flare $300 \mathrm{yrs}$ ago. After the flare the luminosity decreases by factor of $1.7 \times 10^{4}$. During the flare the GC has reached a luminosity of $\sim 2 \cdot 10^{39} \mathrm{erg} / \mathrm{s}$ in $\gamma$-rays (if the electron spectrum during the flare extended up to the $10 \mathrm{TeV}$ energy band).

The observed GeV bump in the GC spectrum is explained by IC scattering of the soft photons on the electrons injected during the flare. The cooling time of electrons with energies about $10 \mathrm{GeV}$ is comparable to the time since the flare. Suppression of the flux above the $\mathrm{GeV}$ energy is explained by the fact that electrons emitting at higher energies have already cooled down. The emission above $10 \mathrm{GeV}$ is mostly due to the electrons injected during the on-going low activity period of the source. Above $10 \mathrm{TeV}$ the emission is suppressed by synchrotron losses which exceed the IC losses in the KleinNishina regime of IC scattering. In order to explain the $\mathrm{TeV}$ spectral shape in $\gamma$-rays the magnetic field should be $\sim 10^{-4} \mathrm{G}$.

The inverse Compton model of the $\mathrm{GeV}$ bump of the spectrum could be verified through the variability properties of the signal. If the $\mathrm{GeV}$ bump is really due to the electrons which were injected 300 years ago during a major flare of the source, gradual cooling of these electrons should lead to the displacement of the bump toward lower energies in the future. A noticeable displacement should occur already on the $10 \mathrm{yr}$ time scale. The $1-10 \mathrm{GeV}$ flux is expected to decrease by $\sim 5-10 \%$ on this time scale. The sensitivity of Fermi/LAT is marginally sufficient for the detection of such decrease.

\subsection{Hadronic model}

The authors of [8] and [7] developed a model in which the GC Fermi/HESS spectrum is explained by hadronic radiation from relativistic protons that diffuse by $\sim 5 \mathrm{pc}$ away from the central source. Data above $100 \mathrm{MeV}$ are well described by the model for different sets of parameters (density profile of surrounding medium, characteristics of the diffusion coefficient, injection rate history). A generic feature of the hadronic models is low-energy cut-off in the spectrum at $100 \mathrm{MeV}$ at pion production threshold, see right panel of Fig. 4. The photon spectrum for the parameters considered by [7] is shown in this figure with thin dotted line. The observational data presented here do not indicate the presence of a cut off in the spectrum around $100 \mathrm{MeV}$. The absence of the cutoff rules out a pure hadronic model of the source spectrum. 
However, a straightforward modification of the hadronic model which takes into account the presence of high-energy electrons along with high-energy protons makes the model consistent with the data. Sufficiently strong Bremsstrahlung and/or IC emission from highenergy electrons in the $100 \mathrm{MeV}$ energy band might "wash out" the pion production threshold feature in the source spectrum. This is illustrated in the right panel of Fig. 4. The electron Bremsstrahlung component of the spectrum, shown by dashed line, is produced by electrons with a cutoff powerlaw injection spectrum with the slope -2 and cut-off at $\sim 1 \mathrm{GeV}$. The injected electrons are cooled by the ionization and bremsstrahlung losses. This results in the production of a break in the electron and Bremsstrahlung spectra at the energy below $100 \mathrm{MeV}$. The high-energy cut-off in the electron spectrum is necessary to avoid the dominance of the Bremsstrahlung emission in the GeV-TeV energy band. The Bremsstrahlung spectrum is normalized to fit the $60-100 \mathrm{MeV}$ (lowest energy) data point. The inverse Compton emission, shown by the dot-dashed line, is sub-dominant at $\lesssim 30 \mathrm{GeV}$ (see e.g. discussion in [7]). The model fit shown in the right panel of Fig. 4 has a ratio of proton to electron densities $n_{p} / n_{e} \gtrsim$ 50 , consistent with values discussed in [7].

\section{Conclusions}

We have presented the results of $\sim 6$ years of Fermi/LAT observations of the Galactic Center. We have shown that the spatial morphology of the GC source is consistent with a point-like source. The $3 \sigma$ upper limit on the radius of the source was found to be $\sim 0.13^{\circ}$ at energies $\gtrsim 10 \mathrm{GeV}$ and $\sim 0.22^{\circ}$ at energies $3-10 \mathrm{GeV}$.

The spectrum of the source presented in this work in the $60-300 \mathrm{MeV}$ energy band does not show any evidence for low-energy cut-off, which is expected in pure hadronic models of the Galactic Center emission.

The MeV-TeV band spectrum of GC is well modelled within a leptonic model in which the GeV "bump" in the spectrum is produced by the IC emission from electrons injected during the strong flare from the GC which happened $\sim 300$ yrs ago. The model has a testable prediction of a decrease of the $1-10 \mathrm{GeV}$ flux by $5-10 \%$ on a $\sim 10$ yrs timescale.

The hadronic models $[7,8]$ are in tension with the observable data. However, we have shown that the inclusion of the electron Bremsstrahlung emission increases the flux below $\sim 100 \mathrm{MeV}$ and removes the tension. We conclude that the presence of a leptonic component in the cosmic rays near the GC is unavoidably required in order to match the predictions of the models with the data.

\section{References}

[1] M.R. Morris, L. Meyer, A.M. Ghez, Research in Astronomy and Astrophysics 12, 995 (2012), 1207.6755

[2] D. Malyshev, M. Chernyakova, A. Neronov, and R. Walter. ArXiv e-prints (2015), 1503.05120

[3] J.R. Mattox, D.L. Bertsch, J. Chiang, B.L. Dingus, S.W. Digel, J.A. Esposito, J.M. Fierro, R.C. Hartman, S.D. Hunter, G. Kanbach, ApJ 461, 396 (1996)

[4] P.L. Nolan, A.A. Abdo, M. Ackermann, M. Ajello, A. Allafort, E. Antolini, W.B. Atwood, M. Axelsson, L. Baldini, J. Ballet, et al., ApJS 199, 31 (2012), 1108.1435

[5] M. Ackermann, M. Ajello, A. Albert, A. Allafort, S. Zimmer. ApJS 203, 4 (2012), 1206. 1896

[6] G. Ponti, M.R. Morris, R. Terrier, A. Goldwurm. Traces of Past Activity in the Galactic Centre, in Cosmic Rays in Star-Forming Environments, edited by, D.F. Torres and O. Reimer (2013), Vol. 34 of Astrophysics and Space Science Proceedings, p. 331, 1210.3304

[7] M. Chernyakova, D. Malyshev, F.A. Aharonian, R.M. Crocker, D.I. Jones, ApJ 726, 60 (2011), 1009. 2630

[8] F. Aharonian, A. Neronov, Ap\&SS 300, 255 (2005) 